\title{
The Practical Stabilization for a Class of Networked Systems with Actuator Saturation and Input Additive Disturbances
}

\author{
Dongyan Chen, Shanqiang Li, and Yujing Shi \\ Department of Applied Mathematics, Harbin University of Science and Technology, Harbin 150080, China \\ Correspondence should be addressed to Dongyan Chen, dychen_2004@yahoo.com.cn
}

Received 28 May 2011; Revised 9 August 2011; Accepted 17 August 2011

Academic Editor: Yurong Liu

Copyright (c) 2012 Dongyan Chen et al. This is an open access article distributed under the Creative Commons Attribution License, which permits unrestricted use, distribution, and reproduction in any medium, provided the original work is properly cited.

The practical stabilization problem is investigated for a class of linear systems with actuator saturation and input additive disturbances. Firstly, the case of the input additive disturbance being a bounded constant and a variety of different situations of system matrices are studied for the three-dimensional linear system with actuator saturation, respectively. By applying the Riccati equation approach and designing the linear state feedback control law, sufficient conditions are established to guarantee the semiglobal practical stabilization or oscillation for the addressed system. Secondly, for the case of the input additive disturbances being time-varying functions, a more general class of systems with actuator saturation is investigated. By employing the Riccati equation approach, a low-and-high-gain linear state feedback control law is designed to guarantee the global or semiglobal practical stabilization for the closed-loop systems.

\section{Introduction}

Actuator saturation (control saturation), as a common and typical nonlinear constraint for control systems, is often encountered in various industrial systems, especially in many physical-controlled systems with magnitude limitation in the input. In general, linear systems can be completely controlled by using the linear state feedback, and the semiglobal (or local) stabilization can be achieved [1-3]. Linear systems with actuator saturation are also a special class of nonlinear systems [4]. The occurrence of actuator saturation inevitably affects the control systems performance and may even result in the instability of the controlled systems. Consequently, the actuator saturation has attracted significant attention, and a variety of approaches have been developed in the literature with respect to various types of systems [5-13]. Specifically, the problems of the global (or semiglobal) asymptotic stabilization, the attraction domain estimation, and the practical stabilization have been extensively investigated. 
When the dimension of the integrator is greater than or equal to 3 , the linear systems with input saturation cannot be stabilized by using the linear state feedback control law, so the global asymptotic stabilization of the system cannot be attained. However, if all eigenvalues of the open-loop system have negative real parts, the global asymptotic stabilization for the addressed system can be guaranteed by designing a globally stable boundary control law. Accordingly, the exponentially semiglobal stabilization problems have been widely studied in [1-3] by designing a linear state feedback control law. By employing the linear matrix inequality (LMI) technique, much work has been done in finding the condition of invariant set and the estimation of attraction domain, see for example, [14-18]. Very recently, networked control systems (NCSs) have attracted considerable attention due to their successful applications in a wide range of areas with the advantage of decreasing the installation cost and implementation difficulties [19-27]. It should be pointed out that, in most related literature concerning the actuator saturation problems, the networked systems with actuator saturation and input additive disturbances have not been thoroughly studied yet.

On the other hand, the study of the linear systems with actuator saturation also covers the practical stabilization problem, that is, a controller is designed such that the trajectories of closed-loop system can enter into an arbitrarily small prescribed neighborhood of the origin in finite time and remain thereafter. The practical stabilization problems have been gaining an increasing research interest, and many important results have been reported, see, for example, [28-30]. To mention a few, the problems of global practical stabilization for planar linear systems have been studied $[28,29]$ in the presence of actuator saturation and input additive disturbances. By tuning the value of the parameter and designing a parameterized linear state feedback law, sufficient conditions have been established such that all trajectories of the closed-loop systems approach to an arbitrarily small neighborhood of the origin in a finite time and remain thereafter. Moreover, the global stabilization problem has also been studied in [30] for a class of second-order switched systems with input saturation. It is worth mentioning that, because of the mathematical complexity and computational difficulty, the corresponding results for general multidimensional systems with actuator saturation and input additive perturbations have been not reported. By designing the lowand-high-gain and the scheduled low-and-high-gain control laws, the semiglobal asymptotic stabilization problems have been investigated in [31, 32] for general multidimensional linear systems. The feedback control law has been designed to deal with the matched uncertainties and input additive disturbances. However, strict assumptions on the input additive uncertainties and disturbances have been imposed on the systems. It is, therefore, our aim to address the multidimensional systems with general constraints on the input additive disturbances.

Motivated by the above discussion, we aim to investigate the practical stabilization problem for three-dimensional (multidimensional) system with actuator saturation and input additive disturbances. By employing the Riccati equation approach, the linear state feedback control law is designed to guarantee the practical stabilization for the system with actuator saturation and time-invariant input additive disturbances. Moreover, the practical stabilization of a general multidimensional system with actuator saturation and time-varying input additive disturbances is studied, and the low-and-high-gain linear state feedback control law is synthesized by using the Riccati equation approach. The main contributions of this paper can be highlighted as follows: (1) the practical stabilization problem of threedimensional linear systems is investigated for the first time, which covers actuator saturation as well as input additive disturbances; (2) the low-and-high-gain linear state feedback control 
law is designed for multidimensional system with actuator saturation and time-varying input additive disturbances.

Notations. The notations in this paper are quite standard except where otherwise stated. The superscript " $T$ " stands for matrix transposition; $\mathbb{R}^{n}\left(\mathbb{R}^{n \times m}\right)$ denote, respectively, the $n$ dimensional Euclidean space, the set of all $n \times m$ matrices; the notation $P>0(P \geq 0)$ means that matrix $P$ is real symmetric and positive definite (positive semi-definite); 0 represents a zero matrix with appropriate dimension, respectively; $\|\cdot\|$ denotes the Euclidean norm of a vector and its induced norm of a matrix. In symmetric block matrices or long matrix expressions, we use a star " $*$ " to represent a term that is induced by symmetry. Matrices, if their dimensions are not explicitly stated, are assumed to be compatible for algebraic operations.

\section{The Case of Bounded Constant Input Disturbance}

Consider the following three-dimensional system with actuator saturation and input additive disturbance:

$$
\dot{x}=A x+B \sigma(u+d)
$$

where $x \in \mathbb{R}^{3}$ is the state, $A$ and $B$ are real matrices of appropriate dimensions, $u \in \mathbb{R}$ is the control input, and $d \in \mathbb{R}$ is the disturbance.

The saturation function $\sigma(\cdot)$ is defined as

$$
\sigma(u)=\operatorname{sign}(u) \min \{1,|u|\},
$$

where the notation of "sign" denotes the signum function.

Before proceeding further, we make the following assumptions.

Assumption 2.1. The matrix pair $(A, B)$ is controllable, and the eigenvalues of $A$ have nonpositive real parts.

Assumption 2.2. The input disturbance is bounded, that is, $|d| \leq D$, where $D$ is an arbitrarily large positive scalar.

Based on Assumption 2.1, let $B=\left[\begin{array}{lll}0 & 0 & 1\end{array}\right]^{T}$, matrix $A$ is in one of the following forms:
(a) $A=\left[\begin{array}{lll}0 & 1 & 0 \\ 0 & 0 & 1 \\ 0 & 0 & 0\end{array}\right]$,
(b) $A=\left[\begin{array}{ccc}0 & 1 & 0 \\ 0 & 0 & 1 \\ 0 & 0 & -a\end{array}\right]$,
(c) $A=\left[\begin{array}{ccc}0 & 1 & 0 \\ 0 & 0 & 1 \\ 0 & -a & 0\end{array}\right]$, 

(d) $A=\left[\begin{array}{ccc}0 & 1 & 0 \\ 0 & 0 & 1 \\ -a & 0 & 0\end{array}\right]$,
(e) $A=\left[\begin{array}{ccc}0 & 1 & 0 \\ 0 & 0 & 1 \\ -a & -b & 0\end{array}\right]$,
(f) $A=\left[\begin{array}{ccc}0 & 1 & 0 \\ 0 & 0 & 1 \\ -a & 0 & -b\end{array}\right]$,
(g) $A=\left[\begin{array}{ccc}0 & 1 & 0 \\ 0 & 0 & 1 \\ 0 & -a & -b\end{array}\right]$,
(h) $A=\left[\begin{array}{ccc}0 & 1 & 0 \\ 0 & 0 & 1 \\ -a & -b & -c\end{array}\right]$,

where $a, b$, and $c$ are positive scalars.

These forms of the matrix pair $(A, B)$ correspond to eight different cases for system (2.1). In the following, the problem of practical stabilization for system (2.1) will be investigated by designing the linear state feedback control law. To facilitate our development, we introduce the following definition.

Definition 2.3. Define an ellipsoid $\Omega(P, \rho)$ as follows:

$$
\Omega(P, \rho):=\left\{x \in \mathbb{R}^{3}, x^{T} P x \leq \rho\right\}
$$

where $P \in \mathbb{R}^{3 \times 3}$ is a symmetric positive definite matrix and $\rho$ is a positive scalar.

For system (2.1), the control law under consideration is of the following structure:

$$
u=-\frac{1}{\varepsilon} B^{T} P x
$$

where $\varepsilon \in(0,1]$, and $P$ is a symmetric positive definite matrix to be determined according to the form of $(A, B)$ defined in (2.3).

Lemma 2.4 (see [10]). Letting $u, v \in \mathbb{R}$ with $|v| \leq 1, E_{1}=0$ and $E_{2}=1$, one has

$$
\sigma(u) \in \operatorname{co}\{u, v\}=\operatorname{co}\left\{E_{i} u+E_{i}^{-} v, i=1,2\right\},
$$

where "co" denotes the convex hull and $E_{i}^{-}=1-E_{i}$.

Theorem 2.5. Consider the system (2.1) with $(A, B)$ defined in (2.3) and the control law (2.5).

(i) If A satisfies case ( $g$ ) with $b^{2}>2 a$ or case ( $h$ ) with $b c>a$ in (2.3), then for any given arbitrarily small set $x_{0} \subset \mathbb{R}^{3}$ containing origin in its interior and for any positive scalar $D$, there always exists $\varepsilon^{*} \in(0,1]$ such that, for any $\varepsilon \in\left(0, \varepsilon^{*}\right]$, all trajectories of the closed-loop system will enter into the set $X_{0}$ in finite time and remain thereafter.

(ii) If A satisfies cases (a) or (b) or (c) in (2.3), when the initial state is in some bounded set, then for any given arbitrarily small set $x_{0} \subset \mathbb{R}^{3}$ containing origin in its interior and any positive number $D$, there always exists $\varepsilon^{*} \in(0,1]$, such that, for any $\varepsilon \in\left(0, \varepsilon^{*}\right]$, all 
trajectories of the closed-loop system will enter into the set $x_{0}$ in finite time and remain thereafter.

(iii) If A satisfies cases (d) or (e) or $(f)$ in (2.3), then all trajectories of the closed-loop system are oscillatory.

Proof. Firstly, let us prove (i).

$\left(i_{1}\right)$ Consider the following system

$$
\dot{x}=\left[\begin{array}{ccc}
0 & 1 & 0 \\
0 & 0 & 1 \\
0 & -a & -b
\end{array}\right] x+\left[\begin{array}{l}
0 \\
0 \\
1
\end{array}\right] \operatorname{sat}(u+d),
$$

that is, $A$ satisfies the case $(g)$ with $b^{2}>2 a$ in (2.3).

Let $P$ be the solution to the following algebra Riccati equation (ARE):

$$
A^{T} P+P A-P B B^{T} P=-\left[\begin{array}{ccc}
a^{2} & 0 & 0 \\
0 & b^{2}-2 a & 0 \\
0 & 0 & 1
\end{array}\right] \text {, }
$$

we have

$$
P=\left[\begin{array}{ccc}
a^{2}+a b & a b+a & a \\
* & b^{2}+b & b \\
* & * & 1
\end{array}\right]
$$

Define

$$
\begin{aligned}
& \Omega^{+}:=\left\{x \in \mathbb{R}^{3}, \frac{1}{\varepsilon} B^{T} P x \geq 1+D\right\}, \\
& \Omega^{-}:=\left\{x \in \mathbb{R}^{3}, \frac{1}{\varepsilon} B^{T} P x \leq-1-D\right\}, \\
& \Omega^{0}:=\left\{x \in \mathbb{R}^{3},\left|\frac{1}{\varepsilon} B^{T} P x\right|<1+D\right\} .
\end{aligned}
$$

For any $x \in \Omega^{+}$, we have $B^{T} P x \geq \varepsilon(1+D)$, that is, $a x_{1}+b x_{2}+x_{3} \geq \varepsilon(1+D)$. From (2.7), we obtain

$$
\begin{gathered}
\dot{x}_{1}=x_{2} \\
\dot{x}_{2}=x_{3} \\
\dot{x}_{3}=-a x_{2}-b x_{3}-1 .
\end{gathered}
$$

Furthermore, there is $a \dot{x}_{1}+b \dot{x}_{2}+\dot{x}_{3}=-1$. It means that $a x_{1}+b x_{2}+x_{3}$ will decrease in a constant speed and will be less than $\varepsilon(1+D)$ in finite time. Hence, any trajectory departing 
from $x \in \Omega^{+}$will enter into the interior of $\Omega^{0}$ in finite time. Similarly, any trajectory departing from $x \in \Omega^{-}$will enter into $\Omega^{0}$ in finite time.

We choose the Lyapunov function $V(x)=x^{T} P x$ with $P>0$ being the solution to ARE (2.8). For any $x \in \Omega^{0}$, we have $\left|(1 / \varepsilon(1+D)) B^{T} P x\right|<1$. It follows from Lemma 2.4 that

$$
\text { sat }\left\{\frac{1}{\varepsilon} B^{T} P x-d\right\} \in \operatorname{co}\left\{E_{i}\left(\frac{1}{\varepsilon} B^{T} P x-d\right)+E_{i}^{-} \frac{1}{\varepsilon(1+D)} B^{T} P x, i=1,2\right\} \text {. }
$$

Noticing that $\varepsilon>0$ is a sufficiently small scalar, we have

$$
\begin{aligned}
\dot{V}(x)= & x^{T} P\left[A x-B \operatorname{sat}\left(\frac{1}{\varepsilon} x^{T} P x-d\right)\right]+\left[A x-B \text { sat }\left(\frac{1}{\varepsilon} x^{T} P x-d\right)\right]^{T} P x \\
\leq & \max _{i \in\{1,2\}}\left\{x^{T} P\left[A x-B E_{i}\left(\frac{1}{\varepsilon} B^{T} P x-d\right)+B E_{i}^{-} \frac{1}{\varepsilon(1+D)} B^{T} P x\right]\right. \\
& \left.+\left[A x-B E_{i}\left(\frac{1}{\varepsilon} B^{T} P x-d\right)+B E_{i}^{-} \frac{1}{\varepsilon(1+D)} B^{T} P x\right]^{T} P x\right\} \\
\leq & x^{T}\left[A^{T} P+P A-\frac{2 D}{\varepsilon(1+D)} P B B^{T} P\right] x+2 x^{T} P B d \\
\leq & x^{T}\left[A^{T} P+P A-P B B^{T} P\right] x+2 \varepsilon D(1+D) \\
= & -x^{T}\left[\begin{array}{ccr}
a^{2} & 0 & 0 \\
0 & b^{2}-2 a & 0 \\
0 & 0 & 1
\end{array}\right] x+2 \varepsilon D(1+D) .
\end{aligned}
$$

Define

$$
\Omega_{d}^{7}:=\left\{x \in \mathbb{R}^{3}, x^{T}\left[\begin{array}{ccc}
a^{2} & 0 & 0 \\
0 & b^{2}-2 a & 0 \\
0 & 0 & 1
\end{array}\right] x \leq 2 \varepsilon D(1+D), b^{2}>2 a\right\}
$$

then there exists a scalar $\rho>0$ such that $\Omega(P, \rho)$ is the smallest ellipsoid satisfying $\Omega(P, \rho) \cap$ $\Omega^{0} \supset \Omega_{d}^{7} \cap \Omega^{0}$. Hence, we have $\dot{V}(x)<0$ for all $x \in \Omega(P, \rho) \cap \Omega^{0}$, and $\Omega(P, \rho) \cap \Omega^{0}$ is an invariant set. Hence, any trajectory of the closed-loop system departing from $\Omega(P, \rho) \cap \Omega^{0}$ will enter into $\Omega(P, \rho) \cap \Omega^{0}$ in finite time and remain thereafter. If $\varepsilon \rightarrow 0$, then $\Omega_{d}^{7}$ tends to origin. Therefore, $\Omega(P, \rho) \cap \Omega^{0}$ approaches to the origin.

$\left(\mathrm{i}_{2}\right)$ Assuming that matrix $A$ satisfies the case $(h)$ and $b c>a$, then the system (2.1) can be written as follows:

$$
\dot{x}=\left[\begin{array}{ccc}
0 & 1 & 0 \\
0 & 0 & 1 \\
-a & -b & -c
\end{array}\right] x+\left[\begin{array}{l}
0 \\
0 \\
1
\end{array}\right] \operatorname{sat}(u+d)
$$


It follows from $b c>a$ that $A$ is stable. Then there exists $P>0$ satisfying

$$
A^{T} P+P A=-I_{3}
$$

When $x \in \Omega^{+}$, noting that $B^{T} P x \geq \varepsilon(1+D)$, we have sat $(u+d)=-1$ and

$$
\dot{V}(x)=x^{T}\left(P A+A^{T} P\right) x-2 x^{T} P B \leq-x^{T} x-2 \varepsilon(1+\mathrm{D})<0 .
$$

Similarly, for any $x \in \Omega^{-}$, it is easy to obtain $\dot{V}(x)<0$.

When $x \in \Omega^{0}$, considering $\left|(1 / \varepsilon(1+D)) B^{T} P x\right|<1$ and noticing that $\varepsilon>0$ is a sufficiently small scalar, we have

$$
\begin{aligned}
\dot{V}(x) & =x^{T}\left(P A+A^{T} P\right) x-\frac{2}{\varepsilon} x^{T} P B B^{T} P x+2 \varepsilon D(1+D) \\
& <-x^{T} x+2 \varepsilon D(1+D)
\end{aligned}
$$

Set

$$
\Omega_{d}^{8}:=\left\{x \in \mathbb{R}^{3},\|x\|^{2} \leq 2 \varepsilon D(1+D)\right\} .
$$

Then there exists a scalar $\rho>0$ such that $\Omega(P, \rho)$ is the smallest ellipsoid which satisfies $\Omega(P, \rho) \cap \Omega^{0} \supset \Omega_{d}^{8} \cap \Omega^{0}$. Now, we can conclude that $\dot{V}(x)<0$ holds for all $x \notin \Omega(P, \rho) \cap \Omega^{0}$. Therefore, all trajectories will enter into $\Omega(P, \rho) \cap \Omega^{0}$ in finite time. When $\varepsilon \rightarrow 0, \Omega(P, \rho) \cap \Omega^{0}$ approaches to the origin.

Secondly, let us prove (ii).

(ii 1 ) Consider the following system:

$$
\dot{x}=\left[\begin{array}{lll}
0 & 1 & 0 \\
0 & 0 & 1 \\
0 & 0 & 0
\end{array}\right] x+\left[\begin{array}{l}
0 \\
0 \\
1
\end{array}\right] \operatorname{sat}(u+d)
$$

that is, $A$ satisfies the case (a).

In this case, we choose the matrix $P$ to be the solution to the following ARE:

$$
A^{T} P+P A-\frac{2}{\varepsilon(1+D)} P B B^{T} P=-\frac{1}{\varepsilon(1+D)}\left[\begin{array}{ccc}
1 & 0 & 0 \\
0 & 2 & 0 \\
0 & 0 & \frac{1-8 \varepsilon(1+D)}{4}
\end{array}\right]
$$


where $0<\varepsilon<1 /(8(1+D))$. Then, we obtain

$$
P=\left[\begin{array}{ccc}
\frac{\sqrt{2}}{\varepsilon(1+D)} & \frac{1}{2 \varepsilon(1+D)} & \frac{\sqrt{2}}{2} \\
* & \frac{\sqrt{2}\left(\frac{\sqrt{1-\varepsilon(1+D)}}{2(1+D)}\right)}{2} & 1 \\
* & * & \frac{\sqrt{2}}{4}
\end{array}\right] .
$$

For any $x \in \Omega^{+}, B^{T} P x \geq \varepsilon(1+D)$ and $\operatorname{sat}(u+d)=-1$. The system (2.20) reduces to

$$
\dot{x}=\left[\begin{array}{lll}
0 & 1 & 0 \\
0 & 0 & 1 \\
0 & 0 & 0
\end{array}\right] x-\left[\begin{array}{l}
0 \\
0 \\
1
\end{array}\right] .
$$

Hence, the solution to (2.23) can be described as:

$$
\begin{gathered}
x_{1}=-\frac{1}{6} t^{3}+\frac{1}{2} x_{03} t^{2}+x_{02} t+x_{01}, \\
x_{2}=-\frac{1}{2} t^{2}+x_{03} t+x_{02}, \\
x_{3}=-t+x_{03},
\end{gathered}
$$

where $x(0)=\left[\begin{array}{lll}x_{01} & x_{02} & x_{03}\end{array}\right]^{T}$ is the initial state.

By noting $B^{T} P x \geq \varepsilon(1+D)$, we have $(\sqrt{2} / 2) x_{1}+x_{2}+(\sqrt{2} / 4) x_{3} \geq \varepsilon(1+D)$. Furthermore, we obtain

$$
\begin{aligned}
B^{T} P \dot{x} & =\frac{\sqrt{2}}{2} \dot{x}_{1}+\dot{x}_{2}+\frac{\sqrt{2}}{4} \dot{x}_{3} \\
& =-\frac{\sqrt{2}}{4} t^{2}-\left(1-\frac{\sqrt{2}}{2} x_{03}\right) t-\frac{\sqrt{2}}{4}+x_{03}+\frac{\sqrt{2}}{2} x_{02}
\end{aligned}
$$

If $B^{T} P \dot{x}<0$, then any trajectory departing from $\Omega^{+}$will enter into $\Omega^{0}$ in finite time. Let

$$
\frac{\sqrt{2}}{4} t^{2}+\left(1-\frac{\sqrt{2}}{2} x_{03}\right) t+\frac{\sqrt{2}}{4}-x_{03}-\frac{\sqrt{2}}{2} x_{02}>0
$$

For $t>0$, the discriminant of quadratic inequality is less than zero, then it is accessible to the conditions of the initial point set. 
Define

$$
X_{1}^{+}:=\left\{x=\left[\begin{array}{lll}
x_{1} & x_{2} & x_{3}
\end{array}\right]^{T} \in \Omega^{+}: x_{1} \in \mathbb{R}, \frac{1}{2} x_{3}^{2}+x_{2}+\frac{1}{2}<0\right\}
$$

then any trajectory departed from $X_{1}^{+}$will enter into $\Omega^{0}$ in finite time and remain thereafter.

Similarly, when $x \in \Omega^{-}$, a set of initial points can also be found such that any trajectory departing from the set will enter into $\Omega^{0}$ in finite time and remain thereafter. After some algebraic manipulations, the initial set of points can be expressed as follows:

$$
X_{1}^{-}:=\left\{x=\left[\begin{array}{lll}
x_{1} & x_{2} & x_{3}
\end{array}\right]^{T} \in \Omega^{-}: x_{1} \in \mathbb{R}, \frac{1}{2} x_{3}^{2}-x_{2}+\frac{1}{2}<0\right\} .
$$

For any $x \in \Omega^{0},\left|(1 / \varepsilon(1+D)) B^{T} P x\right|<1$, we have

$$
\begin{aligned}
\dot{V}(x) \leq & x^{T}\left[P A+A^{T} P-\frac{2}{\varepsilon(1+D)} P B B^{T} P\right] x+2 \varepsilon D(1+D) \\
& -\frac{1}{\varepsilon(1+D)} x^{T}\left[\begin{array}{ccc}
1 & 0 & 0 \\
0 & 1 & 0 \\
0 & 0 & \frac{1-8 \varepsilon(1+D)}{4}
\end{array}\right] x+2 \varepsilon D(1+D) .
\end{aligned}
$$

Let

$$
\Omega_{d}^{1}:=\left\{x \in \mathbb{R}^{3}, x^{T}\left[\begin{array}{ccc}
1 & 0 & 0 \\
0 & 1 & 0 \\
0 & 0 & \frac{1-8 \varepsilon(1+D)}{4}
\end{array}\right] x \leq 2 \varepsilon^{2} D(1+D)^{2}, \varepsilon<\frac{1}{8(1+D)}\right\} .
$$

Then there exists a scalar $\rho>0$ such that $\Omega(P, \rho)$ is the smallest ellipsoid which satisfies $\Omega(P, \rho) \cap \Omega^{0} \supset \Omega_{d}^{1} \cap \Omega^{0}$. Now, we can conclude that $\dot{V}(x)<0$ holds for all $x \in \Omega(P, \rho) \cap \Omega^{0}$. So $\Omega(P, \rho) \cap \Omega^{0}$ is an invariant set. For the initial state $x \notin \Omega(P, \rho) \cap \Omega^{0}$, any trajectory of the system departing from $x \in X_{1}^{+} \cup X_{1}^{-} \cup\left(\Omega^{0} \backslash \Omega(P, \rho)\right)$ will enter into $\Omega(P, \rho) \cap \Omega^{0}$ in finite time and remain thereafter. When $\varepsilon \rightarrow 0, \Omega(P, \rho) \cap \Omega^{0}$ approaches to the origin.

$\left(\mathrm{ii}_{2}\right)$ Consider the following system:

$$
\dot{x}=\left[\begin{array}{ccc}
0 & 1 & 0 \\
0 & 0 & 1 \\
0 & 0 & -a
\end{array}\right] x+\left[\begin{array}{l}
0 \\
0 \\
1
\end{array}\right] \operatorname{sat}(u+d)
$$

that is, $A$ satisfies the case (b). 
In this case, we choose the matrix $P$ to be the solution to the following ARE:

$$
A^{T} P+P A-\frac{2}{\varepsilon} P B B^{T} P=-\frac{2}{\varepsilon}\left[\begin{array}{ccc}
\frac{1}{16} a^{4} & 0 & 0 \\
0 & \frac{1}{2} a^{2} & 0 \\
0 & 0 & 1
\end{array}\right] .
$$

Then, we obtain

$$
P=\left[\begin{array}{ccc}
\frac{a^{3}}{2 \varepsilon} & \frac{a^{2}}{2 \varepsilon} & \frac{1}{4} a^{2} \\
* & \frac{2}{\varepsilon} a-\frac{1}{4} a^{2} & a \\
* & * & 1
\end{array}\right],
$$

where, if $a>6$, then $\varepsilon<6 / a$, and, if $0<a \leq 6$, then $\varepsilon \in(0,1]$.

Define

$$
\begin{aligned}
& X_{2}^{+}:=\left\{x=\left[\begin{array}{lll}
x_{1} & x_{2} & x_{3}
\end{array}\right]^{T} \in \Omega^{+}: x_{1} \in \mathbb{R}, x_{2} \leq \frac{4}{a^{2}}, x_{3} \geq-\frac{1}{a}\right\}, \\
& X_{2}^{-}:=\left\{x=\left[\begin{array}{lll}
x_{1} & x_{2} & x_{3}
\end{array}\right]^{T} \in \Omega^{-}: x_{1} \in \mathbb{R}, x_{2} \geq \frac{4}{a^{2}}, x_{3} \leq-\frac{1}{a}\right\}, \\
& \Omega_{d}^{2}:=\left\{x \in \mathbb{R}^{3}, x^{T}\left[\begin{array}{rrr}
\frac{1}{16} a^{4} & 0 & 0 \\
0 & \frac{1}{2} a^{2} & 0 \\
0 & 0 & 1
\end{array}\right] x \leq \varepsilon^{2} D(1+D), \varepsilon<\frac{6}{a}, a>6, \varepsilon \in(0,1], 0<a \leq 6\right\} .
\end{aligned}
$$

Then there exists a scalar $\rho>0$ such that $\Omega(P, \rho)$ is the smallest ellipsoid which satisfies $\Omega(P, \rho) \cap \Omega^{0} \supset \Omega_{d}^{2} \cap \Omega^{0}$. Therefore, $\dot{V}(x)<0$ holds for all $x \in \Omega(P, \rho) \cap \Omega^{0}$. Hence $\Omega(P, \rho) \cap \Omega^{0}$ is an invariant set. For the initial state $x$ outside $\Omega(P, \rho) \cap \Omega^{0}$, any trajectory departing from $x \in X_{2}^{+} \cup X_{2}^{-} \cup\left(\Omega^{0} \backslash \Omega(P, \rho)\right)$ will enter into $\Omega(P, \rho) \cap \Omega^{0}$ in finite time and remain thereafter. Moreover, when $\varepsilon \rightarrow 0, \Omega(P, \rho) \cap \Omega^{0}$ approaches to the origin.

$\left(\mathrm{ii}_{3}\right)$ Consider the following system:

$$
\dot{x}=\left[\begin{array}{ccc}
0 & 1 & 0 \\
0 & 0 & 1 \\
0 & -a & 0
\end{array}\right] x+\left[\begin{array}{l}
0 \\
0 \\
1
\end{array}\right] \operatorname{sat}(u+d),
$$

that is, $A$ satisfies the case $(c)$. 
In this case, we choose the matrix $P$ to be the solution to the following ARE:

$$
A^{T} P+P A-\frac{2}{\varepsilon} P B B^{T} P=-\frac{1}{\varepsilon}\left[\begin{array}{ccc}
2 a^{2} & 0 & 0 \\
0 & \frac{1-6 \varepsilon^{2} a}{2 \varepsilon^{3}} & 0 \\
0 & 0 & 1
\end{array}\right] .
$$

Then, we obtain

$$
P=\left[\begin{array}{ccc}
a^{2}+\frac{a}{\varepsilon^{2}} & \frac{2 a}{\varepsilon} & a \\
* & \frac{1}{\varepsilon^{2}} & \frac{1}{2 \varepsilon} \\
* & * & 1
\end{array}\right],
$$

where, if $a>1 / 6$, then $\varepsilon<\sqrt{(1 / 6) a}$, and, if $0<a \leq 1 / 6$, then $\varepsilon \in(0,1]$.

Set

$$
\begin{aligned}
& X_{3}^{+}:=\left\{x=\left[\begin{array}{lll}
x_{1} & x_{2} & x_{3}
\end{array}\right]^{T} \in \Omega^{+}: x_{1} \in \mathbb{R}, \sqrt{a} x_{2}+x_{3}<2 \varepsilon-\frac{1}{\sqrt{a}}\right\}, \\
& X_{3}^{-}:=\left\{x=\left[\begin{array}{lll}
x_{1} & x_{2} & x_{3}
\end{array}\right]^{T} \in \Omega^{-}: x_{1} \in \mathbb{R}, \sqrt{a} x_{2}+x_{3}<2 \varepsilon+\frac{1}{\sqrt{a}}\right\}, \\
& \Omega_{d}^{3}:=\left\{x \in \mathbb{R}^{3}, x^{T}\left[\begin{array}{ccc}
2 a^{2} & 0 & 0 \\
0 & \frac{1-6 \varepsilon^{2} a}{2 \varepsilon^{3}} & 0 \\
0 & 0 & 1
\end{array}\right] x \leq 2 \varepsilon^{2} D(1+D),\right. \\
&\left.\varepsilon<\sqrt{\frac{1}{6}} a, a>\frac{1}{6}, \varepsilon \in(0,1], 0<a \leq \frac{1}{6}\right\} .
\end{aligned}
$$

Then, there exists a scalar $\rho>0$ such that $\Omega(P, \rho)$ is the smallest ellipsoid which satisfies $\Omega(P, \rho) \cap \Omega^{0} \supset \Omega_{d}^{2} \cap \Omega^{0}$. Therefore, $\dot{V}(x)<0$ is true for all $x \in \Omega(P, \rho) \cap \Omega^{0}$. Hence, $\Omega(P, \rho) \cap \Omega^{0}$ is an invariant set. For the initial state $x \notin \Omega(P, \rho) \cap \Omega^{0}$, any trajectory of the system departing from $x \in X_{3}^{+} \cup X_{3}^{-} \cup\left(\Omega^{0} \backslash \Omega(P, \rho)\right)$ will enter into $\Omega(P, \rho) \cap \Omega^{0}$ in finite time and remain thereafter. Moreover, when $\varepsilon \rightarrow 0, \Omega(P, \rho) \cap \Omega^{0}$ approaches to the origin.

Finally, let us prove (iii).

Assuming that matrix $A$ satisfies any case among $(d)-(f)$, when the actuator is saturated, all the system trajectories departed from $\Omega^{+}$and $\Omega^{-}$are oscillatory, that is, all the trajectories cannot back into any arbitrarily small set containing the origin in finite time. When the actuator is unsaturated, that is, $x \in \Omega^{0}$. The positive definite symmetric matrix $P$ satisfying certain ARE can be obtained. When $\varepsilon \rightarrow 0$, there exists a small ellipsoid $\Omega_{d}^{i}$ which contains origin as its interior and approaches to the origin such that $\dot{V}(x)<0$ for all $x \in \Omega^{0} \backslash \Omega_{d}^{i}$. Furthermore, there exists $\rho>0$ such that $\Omega(P, \rho)$ is the smallest ellipsoid which 
satisfies $\Omega(P, \rho) \cap \Omega^{0} \supset \Omega_{d}^{i} \cap \Omega^{0}$, that is, $x \notin \Omega(P, \rho) \cap \Omega^{0}$, the trajectory only starting from $\Omega^{0} \backslash \Omega(P, \rho)$ can enter into $\Omega(P, \rho) \cap \Omega^{0}$ in finite time.

Based on (i)-(iii), the proof of this theorem is now complete.

Remark 2.6. It should be pointed out that, because of the mathematical complexity and computational difficulty, almost all papers concerning the actuator saturation and input additive disturbances have considered the two-dimensional systems. In this paper, we make the first attempt to investigate the practical stabilization problem for three-dimensional system with actuator saturation and input additive disturbances. To the best of our knowledge, the research topic addressed in this paper is new and meaningful. The above attempts distinguish our research results from the existing ones.

Remark 2.7. The sufficient conditions are established in Theorem 2.5 for three-dimensional system with actuator saturation and input additive disturbance. By applying the Riccati equation approach, the linear state feedback control law is designed such that the semiglobal practical stabilization can be guaranteed. It should be pointed out that the simultaneous consideration of actuator saturation and input additive disturbance leads to essential difficulties in the technical development, and the corresponding derivations are nontrivial. More specifically, some constructive strategies are introduced to facilitate the derivation of the main results.

\section{The Case of Time-Varying Uncertain Input Disturbance}

In this section, we investigate a more general class of systems with actuator saturation and time-varying disturbance input. A low-and-high-gain is designed to guarantee the global or semiglobal practical stabilization for the closed-loop systems.

Consider the following linear system with actuator saturation and time-varying disturbance input

$$
\dot{x}=A x+B \sigma(u+g(x, t))
$$

where $x \in \mathbb{R}^{n}$ is the state vector, $u \in \mathbb{R}^{m}$ is the control input, $g(x, t): \mathbb{R}^{n} \times \mathbb{R} \rightarrow \mathbb{R}^{m}$ represents the time-varying uncertainty, and $A$ and $B$ are known real matrices with appropriate dimensions. $\sigma(\cdot): \mathbb{R}^{m} \rightarrow \mathbb{R}^{m}$ is the standard saturated function, that is,

$$
\sigma(s)=\left[\begin{array}{llll}
\sigma_{1}\left(s_{1}\right) & \sigma_{2}\left(s_{2}\right) & \cdots & \sigma_{m}\left(s_{m}\right)
\end{array}\right]^{T}, \quad \sigma_{i}\left(s_{i}\right)=\operatorname{sign}\left(s_{i}\right) \min \left\{1,\left|s_{i}\right|\right\}, \quad s \in \mathbb{R}^{m} .
$$

Before proceeding further, we make the following assumptions.

Assumption 3.1. The matrix pair $(A, B)$ is asymptotically null controllable with bounded control, that is, all the eigenvalues of $A$ are in the left half plane (LHP) and $(A, B)$ is stabilizable.

Assumption 3.2. The uncertain element $g(x, t)$ is piecewise continuous in $t$ and locally Lipschitz in $x$ satisfying

$$
\|g(x, t)\| \leq g_{0}(\|x\|)+D_{0}, \quad \forall(x, t) \in \mathbb{R}^{n} \times \mathbb{R}^{+},
$$


where $D_{0}$ is a known positive scalar, and the known function $g_{0}(\cdot): \mathbb{R}^{+} \rightarrow \mathbb{R}^{+}$is local Lipschitz and satisfies $g_{0}(\|x\|) \leq L\|x\|$ with $L>0$ being a constant.

Problem 1. For the system (3.1), the purpose of this section is to design a state feedback control law $u=F x$ such that the following requirements are satisfied.

(1) If $D_{0}=0$, the closed-loop system is globally asymptotically stable at the point of $x=0$.

(2) If $D_{0}>0$, the trajectories of the system enter into a given set which contains the origin as an interior point and remain thereafter.

Remark 3.3. As discussed in [31], corresponding to the specific values for $D_{0}$ and $g_{0}$, Problem 1 refers to different names. Specifically, when $g_{0}=0, D_{0}=0$, it is called the globally asymptotical stabilization by state feedback problem. When $g_{0}=0, D_{0}>0$, it is called the global disturbance rejection by state feedback problem. When $g_{0} \neq 0, D_{0}=0$, it is called the robust globally asymptotical stabilization by state feedback problem. When $g_{0} \neq 0, D_{0}>0$, it is called the robust global disturbance rejection by state feedback problem.

For Problem 1, the design of low-and-high gain state feedback control law is composed of three steps. In Steps 1 and 2, the low-gain and high-gain state feedback control law is designed, respectively. Accordingly, the low-and-high-gain control law is designed in Step 3.

Step 1 (low-gain state feedback design). Let $\mathrm{Q}:(0,1] \rightarrow \mathbb{R}^{n \times n}$ be a continuously differentiable and strictly increasing function such that $Q(\varepsilon)$ is symmetric positive definite for each $\varepsilon \in(0,1]$ and $\lim _{\varepsilon \rightarrow 0} Q(\varepsilon)=0$. Consider the following ARE:

$$
A^{T} P(\varepsilon)+P(\varepsilon) A-P(\varepsilon) B B^{T} P(\varepsilon)=-Q(\varepsilon) .
$$

Then, the following lemma is obtained.

Lemma 3.4 (see [32]). Let Assumption 2.1 be satisfied, then, for all $\varepsilon \in(0,1]$, there exists a unique symmetric positive definite matrix $P(\varepsilon)>0$ which solves the $A R E(3.4)$ and $\lim _{\varepsilon \rightarrow 0} P(\varepsilon)=0$.

Now, we construct the low-gain state feedback law as follows:

$$
u_{L}=-B^{T} P(\varepsilon) x,
$$

where $P(\varepsilon)$ is a symmetric positive definite solution to ARE (3.4).

Based on Lemma 3.4, we can conclude that the above low-gain state feedback control law can be arbitrarily small when $\varepsilon$ is arbitrarily small.

Step 2 (high-gain state feedback design). We design the high-gain state feedback control law as follows:

$$
u_{H}=-\rho B^{T} P(\varepsilon) x,
$$

where $\rho \geq 0$ is called high-gain parameter, $\rho=\rho_{0}\left(1+L+D_{0}\right)^{2} / \lambda_{\min }(Q(\varepsilon))$ with $\rho_{0} \geq 0$ being an adjustable constant, $L$ and $D_{0}$ are defined in Assumption 3.2. 
Step 3 (low-and-high-gain state feedback design). Taking the low-gain and high-gain state feedback control law into account, the low-and-high-gain state feedback control law is constructed as follows:

$$
u_{L H}=-(1+\rho) B^{T} P(\varepsilon) x .
$$

Concluded from [32], the state feedback control law (3.7) is one of the optimal control for the linear system (3.1) without any saturation constraint. The following theorem presents a criterion about the solution to Problem 1.

Theorem 3.5. Let Assumption 3.1 be true. There exist positive scalars $\varepsilon$ and $\rho_{0}^{*}$ such that, for all $\varepsilon \in$ $\left(0, \varepsilon^{*}\right]$ and $\rho_{0} \geq \rho_{0}^{*}$, the low-and-high-gain state feedback control law (3.7) is a solution to Problem1.

Proof. To begin with, the cases of saturation and unsaturation are discussed respectively. For convenience, set $P(\varepsilon)=P$ and $Q(\varepsilon)=Q$.

Firstly, we choose the Lyapunov function $V(x)=x^{T} P x$. By calculating the derivative of $V(k)$ along the trajectory of closed-loop system, we have

$$
\begin{aligned}
\dot{V}(x) & =x^{T}\left(A^{T} P+P A\right) x+2 x^{T} P B \sigma\left(-(1+\rho) B^{T} P x+g(x, t)\right) \\
& =-x^{T} Q x+x^{T} P B B^{T} P x+2 x^{T} P B \sigma\left(-(1+\rho) B^{T} P x+g(x, t)\right) \\
& \leq-x^{T} Q x+2 x^{T} P B B^{T} P x+2 x^{T} P B \sigma\left(-(1+\rho) B^{T} P x+g(x, t)\right) .
\end{aligned}
$$

(1) The actuator is with saturation. Here, it is proved only when $\left(B^{T} P x\right)_{i}<0$, where $(\cdot)_{i}$ denotes the $i$ th element of vector $(\cdot)$.

When $1+\rho \geq\left(-(1+\rho) B^{T} P x\right)_{i} \geq 1+L\|x\|+D_{0}$, we arrive at

$$
\begin{gathered}
\left(-(1+\rho) B^{T} P x+g(x, t)\right)_{i} \geq 1, \quad \forall(x, t) \in \mathbb{R}^{n} \times \mathbb{R}^{+}, \\
\sigma_{i}\left(-(1+\rho) B^{T} P x+g(x, t)\right)=1, \quad i=1,2, \ldots, m, \quad \forall(x, t) \in \mathbb{R}^{n} \times \mathbb{R}^{+}, \\
-1 \leq\left(B^{T} P x\right)_{i} \leq-\frac{1+L\|x\|+D_{0}}{1+\rho} \leq-\frac{1}{1+\rho}<0 .
\end{gathered}
$$

Thus, we get

$$
\left(x^{T} P B\right)_{i}\left(\left(B^{T} P x\right)_{i}+1\right) \leq 0 .
$$

Hence,

$$
x^{T} P B B^{T} P x+2 x^{T} P B H \leq 2 \sum_{i=1}^{m}\left(x^{T} P B\right)_{i}\left(\left(B^{T} P x\right)_{i}+1\right)<0,
$$

where $H=\left[\begin{array}{lll}1 & \cdots & 1\end{array}\right]^{T}$. 
It follows from (3.8) that

$$
\begin{aligned}
\dot{V}(x) & =-x^{T} Q x+x^{T} P B B^{T} P x+2 \sum_{i=1}^{m}\left(x^{T} P B\right)_{i} \\
& \leq-x^{T} Q x+2 \sum_{i=1}^{m}\left(x^{T} P B\right)_{i}\left(\left(B^{T} P x\right)_{i}+1\right) \\
& <-x^{T} Q x<0 .
\end{aligned}
$$

If $\left(B^{T} P x\right)_{i}<-1$, for each $(x, t) \in \mathbb{R}^{n} \times \mathbb{R}^{+}$, it can be easily seen that $\left(-(1+\rho) B^{T} P x+g(x, t)\right)_{i} \geq 1$, that is, $\sigma_{i}\left(-(1+\rho) B^{T} P x+g(x, t)\right)=1(i=1,2, \ldots, m)$. Then, we have

$$
\dot{V}(x)=x^{T}\left(A^{T} P+P A\right) x+2 \sum_{i=1}^{m}\left(x^{T} P B\right)_{i} \leq x^{T}\left(A^{T} P+P A\right) x-2 n<0 .
$$

According to Assumption 3.1, we have $A^{T} P+P A<0$ for any $P>0$.

When $-(1+\rho) \leq\left(-(1+\rho) B^{T} P x\right)_{i} \leq-1-L\|x\|-D_{0}$, we obtain

$$
\begin{gathered}
\left(-(1+\rho) B^{T} P x+g(x, t)\right)_{i} \leq-1, \quad \forall(x, t) \in \mathbb{R}^{n} \times \mathbb{R}^{+}, \\
\sigma_{i}\left(-(1+\rho) B^{T} P x+g(x, t)\right)=1, \quad i=1,2, \ldots, m, \forall(x, t) \in \mathbb{R}^{n} \times \mathbb{R}^{+}, \\
1 \geq\left(\mathrm{B}^{T} P x\right)_{i} \geq \frac{1+L\|x\|+D_{0}}{1+\rho} \geq \frac{1}{1+\rho}>0 .
\end{gathered}
$$

That is $\left(B^{T} P x\right)_{i}>0$. Along the same line of the above proof, we can discuss the cases $0<\left(B^{T} P x\right)_{i} \leq 1$ and $\left(B^{T} P x\right)_{i}>1$, respectively. Hence, we can conclude that $\dot{V}(x)<0$ for $\left(B^{T} P x\right)_{i}>0$.

(2) The actuator is not saturated. When $\left|\left(-(1+\rho) B^{T} P x\right)_{i}+(g(x, t))_{i}\right|<1$, according to (3.8), we have

$$
\begin{aligned}
\dot{V}(x) & =-x^{T} Q x-2 \rho x^{T} P B B^{T} P x+2 x^{T} P B g(x, t) \\
& \leq-x^{T} Q x+2 \sum_{i=1}^{m}(g(x, t))_{i}-\rho\left(B^{T} P x\right)_{i}\left(B^{T} P x\right)_{i} .
\end{aligned}
$$


Next, we discuss the following two cases.

If $\left|\left(\rho B^{T} P x\right)_{i}\right| \geq\left|g_{i}\right|$, by noting $\left(B^{T} P x\right)_{i}\left(-\rho\left(B^{T} P x\right)_{i}+(g(x, t))_{i}\right)<0$, we obtain

$$
\begin{aligned}
\dot{V}(x) & =-x^{T} Q x-2 x^{T} P B\left(-(1+\rho) B^{T} P x+g(x, t)+B^{T} P x\right) \\
& \leq-x^{T} Q x+2 \sum_{i=1}^{m}\left(-\rho\left(B^{T} P x\right)_{i}+(g(x, t))_{i}\right) \\
& \leq-x^{T} Q x \\
& <0 .
\end{aligned}
$$

If $\left|\left(\rho B^{T} P x\right)_{i}\right| \leq\left|g_{i}\right|$, then

$$
\begin{aligned}
\dot{V}(x) & =-x^{T} Q x+2 x^{T} P B g(x, t) \\
& \leq-x^{T} Q x+2 \sum_{i=1}^{m} \frac{\left|g_{i}\right|^{2}}{\rho} \\
& \leq-x^{T} Q x+2 \frac{\left(L\|x\|+D_{0}\right)}{\rho} \\
& \leq-\left(\lambda_{\min }(Q)-\frac{4 L^{2}}{\rho}\right)\|x\|^{2}+\frac{4 D_{0}^{2}}{\rho} .
\end{aligned}
$$

Suppose

$$
W_{0}:=\left\{x \in \mathbb{R}^{n}:\|x\|^{2} \leq \frac{4 D_{0}^{2}}{\rho_{0}\left(1+L+D_{0}\right)^{2}-4 L^{2}}\right\} .
$$

Taking $\rho^{*}=4$, then all trajectories will enter into $W_{0}$ in finite time and remain thereafter for all $\rho_{0} \geq \rho^{*}$. Here, $W_{0}$ is an arbitrarily small set including the origin point, and $\rho_{0}$ is a sufficiently large scalar.

Specifically, it follows from $D_{0}=0$ that $\dot{V}(x)<0$, that is, $x=0$ is globally asymptotically stable. To this end, the proof of this theorem is complete.

Remark 3.6. In this section, the practical stabilization problem is investigated for a general multidimensional system. From a practical point of view, it is more significant to consider the high-dimensional systems. It is worth mentioning that the system under consideration is comprehensive that includes the actuator saturation and the time-varying input disturbances. By using the Riccati equation approach, the low-and-high-gain state feedback control law is designed such that the global or semiglobal practical stabilization for the multidimensional system can be guaranteed. On the other hand, we are now researching into a method for the system with uncertainties, time-delay, and/or input disturbance in more general cases. The corresponding results will appear in the near future. 


\section{Conclusions}

In this paper, we have made an attempt to investigate the practical stabilization problem for a class of system with actuator saturation and input additive disturbances. For the case of the input additive disturbance being bounded constant, the three-dimensional system has been studied where the system matrices satisfy a class of the controllability canonical form. Eight different forms of the system matrices have been discussed. Subsequently, by applying the Riccati equation approach and designing the linear state feedback control law, the sufficient conditions of the semiglobal practical stabilization or oscillation for the addressed systems have been established. For the case when the input additive disturbances are time-varying functions, by using of the Riccati equation approach as well as combining the low-gain linear state feedback and high-gain linear state feedback, a low-high-gain linear state feedback control law has been designed such that the global or semiglobal practical stabilization for a general multidimensional system with actuator saturation can be guaranteed. One of the future research topics would be the extension of the main results obtained in this paper to networked control systems [27, 33-36].

\section{Acknowledgment}

This work was supported by the National Natural Science Foundation of China under Grant 10771047.

\section{References}

[1] A. T. Fuller, ".In-the-large stability of relay and saturating control systems with linear controllers," International Journal of Control, vol. 10, pp. 457-480, 1969.

[2] E. D. Sontag and H. J. Sussmann, "Nonlinear output feedback design for linear systems with saturating controls," in Proceedings of the 29th IEEE Conference on Decision and Control, pp. 3414-3416, Honolulu, Hawaii, USA, December 1990.

[3] Z. Lin and A. Saberi, "Semi-global exponential stabilization of linear systems subject to input saturation via linear feedbacks," Systems E Control Letters, vol. 21, no. 3, pp. 225-239, 1993.

[4] H. K. Khali, Nonlinear Systems, Prentice Hall, 3rd edition, 2002.

[5] T. Hu and Z. Lin, Control Systems with Actuator Saturation: Analysis and Design, Birkhauser, Boston, Mass, USA, 2001.

[6] Z. Lin and A. Saberi, "Semi-global exponential stabilization of linear discrete-time systems subject to input saturation via linear feedbacks," Systems $\mathcal{E}$ Control Letters, vol. 24, no. 2, pp. 125-132, 1995.

[7] V. Kapila and K. Grigoriadis, Actuator Saturation Control, Marcel Dekker, New York, NY, USA, 2002.

[8] S. Tarbouriech, G. Garcia, and A. H. Glattfelder, Advanced Strategies in Control Systems with Input and Output Constraints, vol. 346 of Lecture Notes in Control and Information Sciences, Springer, Berlin, Germany, 2007.

[9] T. Hu and Z. Lin, "Robust stabilization of exponentially unstable linear systems with saturating actuators," in Proceedings of the American Control Conference (ACC'99), pp. 3196-3200, San Diego, Calif, USA, June 1999.

[10] H. Fang and Z. Lin, "Stability analysis for linear systems under state constraints," Institute of Electrical and Electronics Engineers. Transactions on Automatic Control, vol. 49, no. 6, pp. 950-955, 2004.

[11] D. Fu, H. Liu, and B. Zhou, "Semi-global stabilization of a type of input systems with pole assurance," Engineering Journal of Wuhan University, vol. 41, no. 2, pp. 111-115, 2008.

[12] X. Wu and Z. Lin, "Anti-windup in anticipation of actuator saturation," in Proceedings of the 49th IEEE Conference on Decision and Control, pp. 5245-5250, Atlanta, Ga, USA, December 2010.

[13] S. Tarbouriech and F. Gouaisbaut, "Stabilization of quantized linear systems with saturations," in Proceedings of the 49th IEEE Conference on Decision and Control, pp. 3361-3366, Atlanta, Ga, USA, month year. 
[14] T. Hu, A. N. Pitsillides, and Z. Lin, "Null controllability and stabilization of linear systems subject to asymmetric actuator saturation," in Proceedings of the 39th IEEE Confernce on Decision and Control, pp. 3254-3259, Sydney, Australia, December 2000.

[15] Y. Cao, Z. Lin, and D. G. Ward, "An antiwindup approach to enlarging domain of attraction for linear systems subject to actuator saturation," Institute of Electrical and Electronics Engineers. Transactions on Automatic Control, vol. 47, no. 1, pp. 140-145, 2002.

[16] T. Hu and Z. Lin, "Exact characterization of invariant ellipsoids for single input linear systems subject to actuator saturation," Institute of Electrical and Electronics Engineers. Transactions on Automatic Control, vol. 47, no. 1, pp. 164-169, 2002.

[17] T. Hu and Z. Lin, "On the necessity of a recent set invariance condition under actuator saturation," in Proceedings of the American Control Conference, pp. 1210-1215, May 2002.

[18] T. Hu and Z. Lin, "The equivalence of several set invariance conditions under saturation," in Proceedings of the 41st IEEE Conference on Decision and Control, pp. 4146-4147, December 2002.

[19] Z. Wang, D. W. C. Ho, Y. Liu, and X. Liu, "Robust $H_{\infty}$ control for a class of nonlinear discrete timedelay stochastic systems with missing measurements," Automatica, vol. 45, no. 3, pp. 684-691, 2009.

[20] B. Shen, Z. Wang, H. Shu, and G. Wei, “On nonlinear $H_{\infty}$ filtering for discrete-time stochastic systems with missing measurements," Institute of Electrical and Electronics Engineers. Transactions on Automatic Control, vol. 53, no. 9, pp. 2170-2180, 2008.

[21] B. Shen, Z. Wang, H. Shu, and G. Wei, "Robust $H_{\infty}$ finite-horizon filtering with randomly occurred nonlinearities and quantization effects," Automatica, vol. 46, no. 11, pp. 1743-1751, 2010.

[22] Z. Wang, Y. Liu, and X. Liu, " $H_{\infty}$ filtering for uncertain stochastic time-delay systems with sectorbounded nonlinearities," Automatica, vol. 44, no. 5, pp. 1268-1277, 2008.

[23] Z. Wang, G. Wei, and G. Feng, "Reliable $H_{\infty}$ control for discrete-time piecewise linear systems with infinite distributed delays," Automatica, vol. 45, no. 12, pp. 2991-2994, 2009.

[24] Z. Wang, F. Yang, D. W. C. Ho, and X. Liu, "Robust $H_{\infty}$ control for networked systems with random packet losses," IEEE Transactions on Systems, Man, and Cybernetics, vol. 37, no. 4, pp. 916-924, 2007.

[25] Z. Wang, F. Yang, D. W.C. Ho, and X. Liu, "Robust $H_{\infty}$ filtering for stochastic time-delay systems with missing measurements," IEEE Transactions on Signal Processing, vol. 54, no. 7, pp. 2579-2587, 2006.

[26] L. Wu, X. Su, P. Shi, and J. Qiu, "A new approach to stability analysis and stabilization of discrete-time T-S fuzzy time-varying delay systems," IEEE Transactions on Systems, Man, and Cybernetics, vol. 41, no. 1, pp. 273-286, 2011.

[27] H. Dong, Z. Wang, and H. Gao, "Robust $H_{\infty}$ filtering for a class of nonlinear networked systems with multiple stochastic communication delays and packet dropouts," IEEE Transactions on Signal Processing, vol. 58, no. 4, pp. 1957-1966, 2010.

[28] H. Fang and Z. Lin, "Global practical stabilization of planar linear systems in the presence of actuator saturation and input additive disturbance," Institute of Electrical and Electronics Engineers. Transactions on Automatic Control, vol. 51, no. 7, pp. 1177-1184, 2006.

[29] H. Fang and Z. Lin, "A further result on global stabilization of oscillators with bounded delayed input," Institute of Electrical and Electronics Engineers. Transactions on Automatic Control, vol. 51, no. 1, pp. 121-128, 2006.

[30] Y. Song, Y. Q. Gu, and M. Fei, "Stabilization for a class of second-order switched systems with saturation constrains," Journal of Shanghai University, vol. 13, no. 4, pp. 274-275, 2009.

[31] A. Saberi, Z. Lin, and A. R. Teel, "Control of linear systems with saturating actuators," Institute of Electrical and Electronics Engineers. Transactions on Automatic Control, vol. 41, no. 3, pp. 368-378, 1996.

[32] Z. Lin, "Global control of linear systems with saturating actuators," in Proceedings of the 35th IEEE Conference on Decision and Control, pp. 4357-4362, Kobe, Japan, December 1996.

[33] B. Shen, Z. Wang, Y. S. Hung, and G. Chesi, "Distributed $H_{\infty}$ filtering for polynomial nonlinear stochastic systems in sensor networks," IEEE Transactions on Industrial Electronics, vol. 58, no. 5, pp. 1971-1979, 2011.

[34] B. Shen, Z. Wang, and X. Liu, "Bounded $H_{\infty}$ synchronization and state estimation for discrete timevarying stochastic complex networks over a finite horizon," IEEE Transactions on Neural Networks, vol. 22, no. 1, pp. 145-157, 2011.

[35] B. Shen, Z. Wang, and Y. S. Hung, "Distributed Ho-consensus filtering in sensor networks with multiple missing measurements: the finite-horizon case," Automatica, vol. 46, no. 10, pp. 1682-1688, 2010. 
[36] H. Dong, Z. Wang, D. W. C. Ho, and H. Gao, "Variance-constrained $H_{\infty}$ filtering for a class of nonlinear time-varying systems with multiple missing measurements: the finite-horizon case," IEEE Transactions on Signal Processing, vol. 58, no. 5, pp. 2534-2543, 2010. 


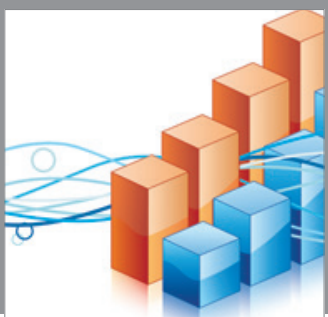

Advances in

Operations Research

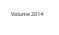

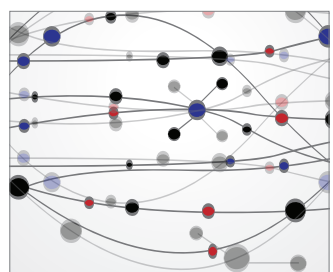

\section{The Scientific} World Journal
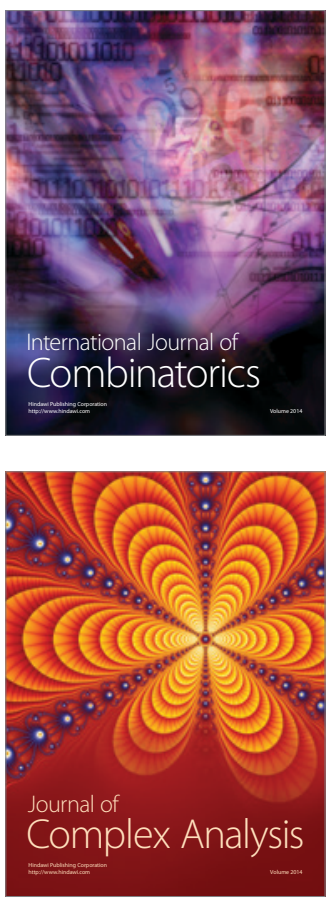

International Journal of

Mathematics and

Mathematical

Sciences
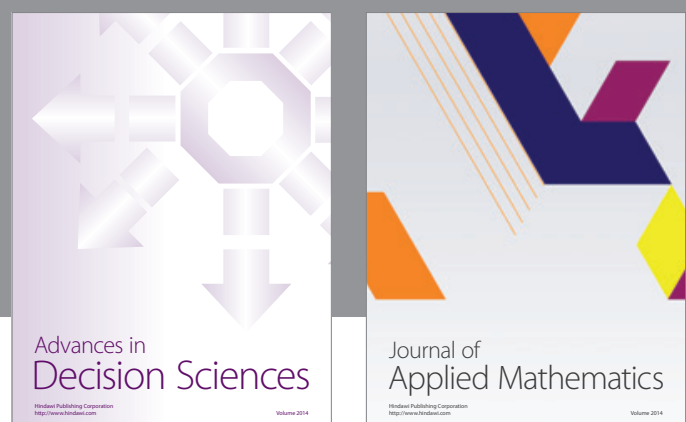

Journal of

Applied Mathematics
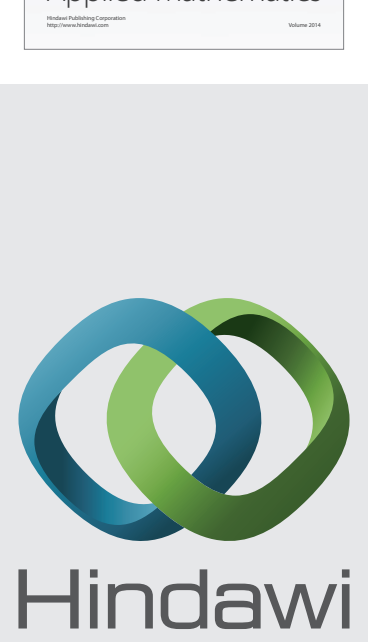

Submit your manuscripts at http://www.hindawi.com
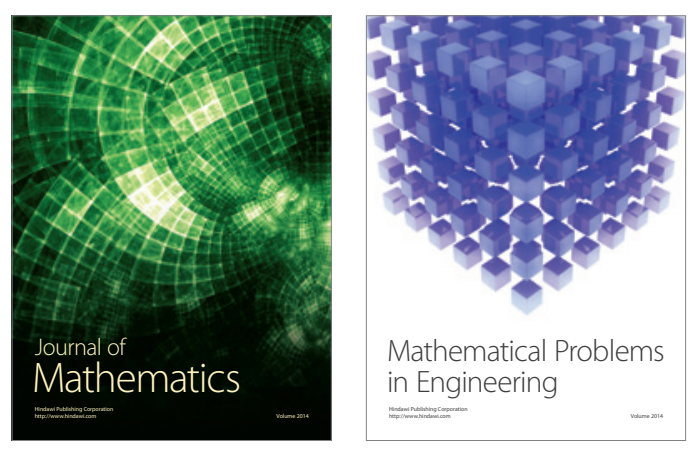

Mathematical Problems in Engineering
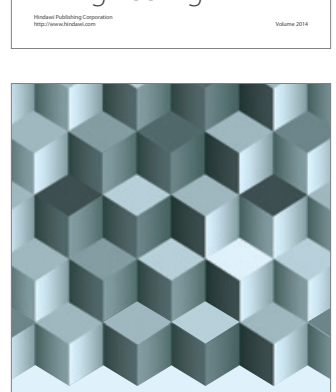

Journal of

Function Spaces
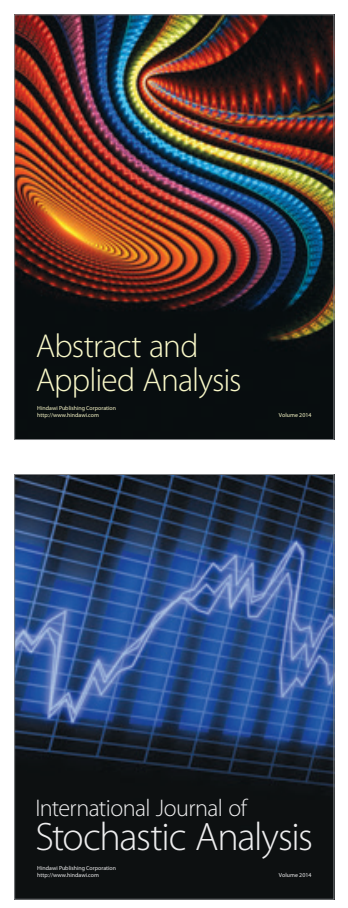

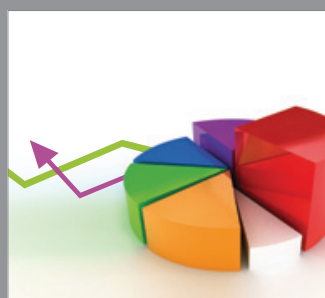

ournal of

Probability and Statistics

Promensencen
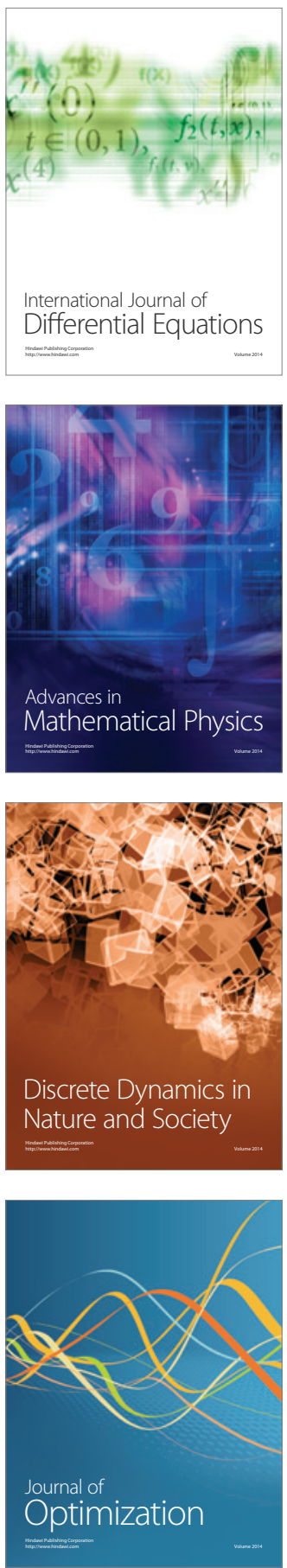